\title{
Implementation of ANPR System based on Image Extraction and Segmentation
}

\author{
Raghuvamsi Machiraju ${ }^{1}$, Gunna Kishore ${ }^{2}$ \\ ${ }^{1,2}$ Computer Science and Engineering, Rise Group of Institutions, Ongole, India
}

\begin{abstract}
This paper discussed on automatic parking system and electronic parking fee collection based on vehicle number plate recognition. The aim of this research is to develop and implement an automatic parking system that will increase convenience and security of the public parking lot as well as collecting parking fee without hassles of using magnetic card. The auto parking system will able to have less interaction of humans and use no magnetic card and its devices. In additions to that, it has parking guidance system that can show and guide user towards a parking space. The system used image processing of recognizing number plates for operation of parking and billing system. Overall, the systems run with pre-programmed controller to make minimum human involvement in parking system and ensure access control in restricted places. This paper presents algorithm technology based method for license plate extraction from car images followed by the segmentation of characters and reorganization and also develop electronics parking fee collection system based on number plate information.
\end{abstract}

Keywords: Automatic parking system, autocorrelation, image processing, mean square error, plate number recognition structural similarity index.

\section{Introduction}

The car license plate recognition identification is an important application in the field of Intelligent Transport System (ITS) and Electronic toll collection (ETC). The objective is to extract and recognize vehicle registration numbers from car images, process the image data finally utilize for access record and prepare electronic bill. Electronic toll collection (ETC) or Electronic Car parking payment is one of the major research topics in intelligent transportation system (ITS) [1]. ETC is an implementation of a road pricing concept in order to create benefits such as increasing the capacity of toll stations, reducing a toll paying time, enhancing the convenience and safety of travelers, and minimizing air pollution and fuel consumption. It enables freeway to parking lot, toll plaza, and bridge, tunnel, and turnpike operators to save on staffing costs while reducing delay for travelers and improve overall traffic performance and parking system. Moreover, monitoring the vehicle traffic and the management of parking areas are the most laborintensive job. Therefore the research on systematic full automatic parking system is proposed. It differs from conventional parking system, no magnetic card is used to record the entry and exit time of. Also, it is designed in such a. way that it has the ability of giving out the information regarding parking free spaces to users before entering the parking spaces.

In the recent years, License Plate Recognition (LPR) are having a wide impact in people"s life as their scope is to improve transportation safety and mobility and to enhance productivity through the use of advanced technologies [1]. This system is useful in many fields and places such as parking lots, private and public entrances, border control, theft and vandalism control. For the past two decades, there have been various studies for number plate recognition in images. Early day's number plate recognition systems employed detection methods based on techniques such as edge analysis [1-3], color analysis [4-5], and the Adaboost training [6]. S.Z. Wang and H.M. Lee [1], D. Zheng et al. [2], and P. Rattanathammawat and T.H. Chalidabhongse [7] proposed an edge analysis method for license plate detection. Ernst [7-9] introduced a face detection method based on local structure patterns computed by the Modified Census Transform (MCT).

License plate recognition applies image processing and character recognition technology to identify vehicles by automatically reading the license plates. Basically, to build this system it consists of some major parts which are vehicle number plate extraction, characters segmentation, and characters recognition [10-13]. Vehicle number plates extraction from the car plate images. Before extracting the number plate, the captured vehicle image should have been converted into binary format [14]. After extracting the number plate, the characters are segmented using vertical and localization on the binary image. Optical Character Recognition (OCR) algorithm is used to recognize the character with condition, the background of the image has no or very little noise [15]. The aim of the system is to recognize the license plate number of car of the parking place by algorithmic and introduce magnetic card less parking and billing activities.

\section{System Overview}

Recognition of any License Plate Recognition system is the effectiveness of its algorithms. Six primary algorithms are used for this License Plate Recognition system

\section{License Plate Localization}

Firstly the car image is captured. Then, the system should extract the number plate of the car alone for the segmentation of character purpose. This plate localization algorithm is based on combining morphological operation sensitive to specific shapes in the input image with a good threshold value by which the license plate is located. A big percentage of localization of License plates is achieved by this algorithm. This variance can further compound the complexity for an 
algorithm to ascertain what area of a vehicle constitutes a license plate and what area is not. Therefore, the algorithm must rule out a vehicle's mirror, headlight, bumper etc. In general, algorithms look for geometric shapes of rectangular proportion. However, since a vehicle can have many rectangular objects on it, further algorithms are needed to validate that the identified object is indeed a license plate. To accomplish this, key components of the algorithm look for characteristics that would indicate that the object is a license plate. The algorithm searches for a similar background color of unified proportion and contrast as a means to differentiate objects on a vehicle. Vehicles are moving objects and their rate of velocity must be accounted for in the algorithm's design. This speed creates further complexity as a license plates image is angularly skewed and subjected to refractory issues from light changes.

\section{License Plate Sizing and Orientation}

Components of algorithms that adjust for the angular skew of the license plate image to accurately sample, correct, and proportionally recalculate to an optimal size.

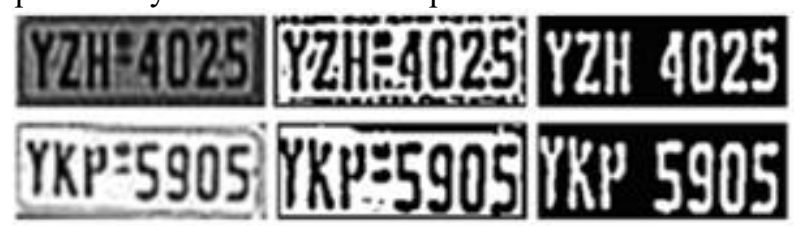

Figure 1: License plate sizing sequence

\section{Characters Segmentation}

Character segmentation is an important step in license plate recognition system. The segmentation of characters in a license plate is performed by using the following steps.

It is very important for the good performance of character segmentation. The preprocessing consists of the determination of plate kind. There are two kinds of license plate in India. One is black characters in yellow background and the other is black characters in white background. The color image is transformed into gray scale image.

\section{EKR799}

Figure 2: Character segmentation

One of the study use Red color algorithm approach [5]. The techniques will search yellow pixel or some that closer to yellow in value from the image. Red pixel is set to 1 and others will be 0 . Then, the recognized image will be converted in binary format for further template matching approach.

\section{Normalization}

The algorithm in regulating the contrast and brightness of the captured license plate image is shown below in Fig. 3 .

\section{EKR799 $\rightarrow$ JEKR799 5EKR799 5EKR7}

\section{Optical Character Recognition (OCR)}

This is the process that identifies individual alpha numeric characters on a license plate. Algorithms also look for characters of equal color and equidistance, with similar font structures to break apart each individual character. This sequential congruency of the characters embodies a characteristic set that is typically uniform, regardless of the type of license plate. Character Segmentation separates each letter or number where it is subsequently processed by optical character recognition (OCR) algorithms. It translates the captured image into an alpha numeric text entry.

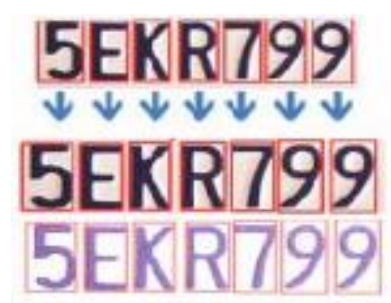

Fig.4 Optical character recognition process

\section{Syntactical /Geometrical Analysis}

Algorithm to verify alpha numeric information and arrangement with a specific rule set. The algorithms operate sequentially with instructions being executed in milliseconds. The successful completion of each algorithm is required before subsequent algorithms can be operational.

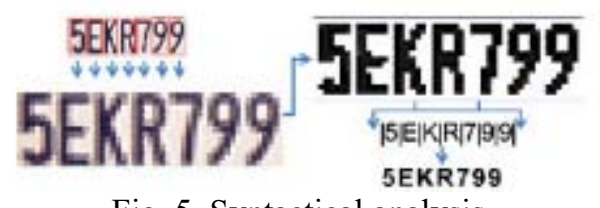

Fig. 5. Syntactical analysis

\section{Image Acquisition \& Plate Number Recognition}

Following flow chart shows the general step of algorithm used for plate number recognition in this project. This algorithm is chosen because it is suitable for number plates. 


\section{International Journal of Science and Research (IJSR) \\ ISSN (Online): 2319-7064}

Index Copernicus Value (2013): 6.14 | Impact Factor (2014): 5.611

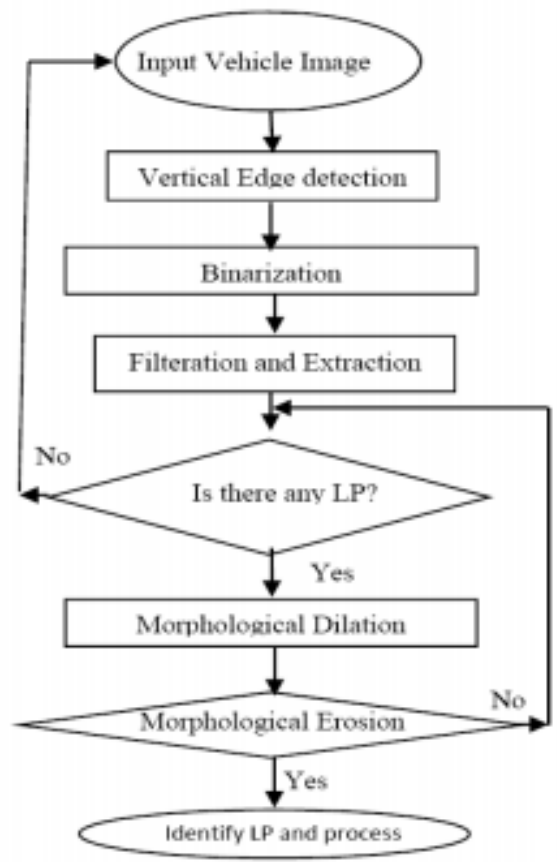

Fig 6. License plate recognition flowchart

\section{RGB to binary conversion}

a. Firstly, the image must be converted to grayscale because the CCD camera captures an RGB image. Also, the conversion is done to increase the processing speed as grayscale image use less memory.

b. The image is resized to $800 \times 600$ pixels.

c. Then, the grayscale image is converted into binary image which is consists of 1 and 0 digitally for each pixel of image.

\section{Image Filtration}

a. Each connected component will be assigned to an integer number from 1 to the total number of adjacent components. $b$. After the connected components have been labeled, their width and height are been calculated. The minimum and maximum width and height of the labeling object (in pixels) are;

- $\quad$ Heightmin $=3$

- $\quad$ Widthmin $=2$

- $\quad$ Heightmax $=70$

- $\quad$ Widthmin $=80$

c. Each connected component that is calculated will be compared to the referenced width and height. As for the height values, only the connected component that had height less than Tmax_h and greater than Tmin_h, will be retained. Otherwise, the object will be removed.

d. As a result, the image after this process only contained the number plate and several objects that had almost same size of the number plate.

e. Then, the area of each connected objects that left are calculated. This process also used connected components techniques. The minimum and maximum area of the number plate are:

\section{- $\quad$ Areamin $=8$}

- $\quad$ Areamin $=1700$

f. The objects that the areas within the reference areas

3. Analysis and Dilation: Then, use dilation (morphological) operation for the resulted image. Dilation adds pixels to the boundaries of objects in an image. So, this process is used to combine the close objects, by using structure element value equals to 8 pixels in 0 degree $\left(0^{\circ}\right)$. Objective of this process is to group the characters and numbers in number plate as a 2 groups.

4. Accurate location of License Plate: If the object remained in the image is only 1 , then the system will assume the object is the plate. But, if the object is more than 1 , eliminate all objects that are too near with the border.

5. Character Recognition: To recognize plate numbers, each character must be analyzed.

a. Binarization of the recognized license plate

b. Segmentation of each character

c. Template matching to recognize license plate numbers

6. Performance Measure:

Component heads identify the different components of your paper and are not topically subordinate to each other. Examples include Acknowledgments and References and, for these, the correct style to use is "Heading 5". Use "figure caption" for your Figure captions, and "table head" for your table title. Run-in heads, such as "Abstract", will require you to apply a style (in this case, italic) in addition to the style provided by the drop down menu to differentiate the head from the text.

There are several ways to recognize the characters. The approaches are; (1) autocorrelation, (2) Mean Square Error (MSE) and (3) Structural Similarity Index (SSI).

\section{Autocorrelation:}

It quantifies the closeness between two images. This coefficient value ranges from -1 to +1 , where the value +1 indicates that the two images are highly correlated and are very close to each other.

$$
r=\frac{\sum_{m} \sum_{n}\left(A_{m n}-A^{-}\right)\left(B_{m n}-B^{-}\right)}{\sqrt{\sum_{m} \sum_{n}\left(A_{m n}-A^{-}\right)^{2}} \sum_{m} \sum_{n}\left(B_{m n}-B^{-}\right)^{2}}
$$

where $A^{-}=\operatorname{mean}^{2}(\mathrm{~A})$, and $B^{-}=\operatorname{mean}^{2}(\mathrm{~B})$,

8. Mean Square Error: Computes the square difference between pixels in two different images and then taken the average over all pixels in the image. An image is perfect reproduction of original image will have an MSE of zero, while an image that differs greatly from the original image will have a large MSE.

$$
M S E=\frac{1}{M N} \mp \sum_{Y=1}^{M} \sum_{x=1}^{M}\left|P_{(x, y)}-Q_{(x, y)}\right|^{2}
$$

Where $\mathrm{M}, \mathrm{N}$ are the dimension of the image, ( ) $\mathrm{x}, \mathrm{y} \mathrm{p}$ is a pixel of the original image and $Q_{(x, y)}$ is the corresponding pixel from the reconstructed image.

9. Structural Similarity Index: Universal quality index models any distortion as a combination of three different factors, namely; (a) loss of correlation, (b) luminance distortion and (c) contrast distortion.

$\mathrm{SSI}=[-1,+1](3)$

The best value 1 is achieved if and only if the two images are similar and -1 if the images are highly not similar.

\section{Proposed Algorithm for Character Recognition}


Since there are two databases that we have developed for easier processes, the recognition must be done in two ways; each one for letters and numbers. The characters are cropped and binarized manually. Each character will be presented by four images. These images are taken from different condition such as from front view, slanted to some angle (not fixed), different weather condition either sunny or rainy day, or character with noises. The mean value of these four images will be counted by using MSE approach.

\section{1. 'Full' Display System}

An LCD display is used to show the working principle of access of barrier gate. If the „FULL ${ }^{\text {ee }}$ is displayed, the system would not process the car image and barrier gate would not operate. Plus, the data acquired from ultrasonic sensors of each parking space is calculated for the calculating free parking spaces.

Where $\mathrm{M}, \mathrm{N}$ are the dimension of the image, is a pixel of the original image and $Q_{(x, y)}$ is the corresponding pixel from the reconstructed image.

\subsection{Structural Similarity Index}

Universal quality index models any distortion as a combination of three different factors, namely; (a) loss of correlation, (b) luminance distortion and (c) contrast distortion. According to equation 3 the best value 1 is achieved if and only if the two images are similar and -1 if the images are highly not similar.

\subsection{Direction System}

The direction system starts when the barrier gate is opened. The database will update the status of the empty parking lots for updating the counter for ,FULL" display system. Then, Matlab is used to calculate the nearest lot that has no vehicle to the user. Afterwards, it will display the direction towards the empty lots to the user using LEDs.

\section{Electronics Payment System}

As mention earlier this composite system will provide the number plate recognition and electron parking bill. At the entry, the vehicle will stop before the entry barrier and its presence is detected by loop sensor. The loop sensor will initiate the camera to capture a picture of the vehicle and the LPR module will analyze the captured picture to recognize the number.

The captured picture together with the recognized number and entry record (entry date \& time) will be stored for parking fee calculation later. Once this is completed, the entry barrier will open to allow the vehicle to enter and park. Another loop sensor after the barrier will close the barrier. The entry station is used to interface with the loop sensors and automatic barriers.

When the leaving vehicle reach the exit booth, it will stop before the barrier and its presence is detected by a loop sensor. This sensor will initiate a picture to be taken and the license plate to be read by the LPR module. The LPR module will match the recognized vehicle number with its own file for the entry time for this particular vehicle. Once the exit and entry record are matched, the system will calculate and display the parking fee is due. When the transaction is complete, the exit barrier will open and the vehicle will leave. For barrier control there is another barrier another loop sensor is implemented.

\section{Conclusion}

In this paper the development of an automatic parking system with license plate recognition, parking lots status and guidance parking system and electronic billing system is successfully implemented. The performance of the developed of algorithms for License Plate Localization and License Plate. Recognition is acceptable range. The developed algorithms accurately localize and recognize in different location of the license plate. Electronic billing system performance is also acceptable and recommended for commercial use.

\section{References}

[1] SherrZheng Wang, HsMian Lee, "Detection and Recognition of License Plate Characters with Different Appearences", in proc. Conf. Intelligent Transportation Systems, vol. 2, pp. 979-984, 2003.

[2] Younghyun Lee1, Taeyup Song, Bonhwa Ku1, Seoungseon Jeon, David K. Han, Hanseok Ko "License Plate Detection using Local Structure Patterns" in proc. Seventh IEEE International Conference on Advanced Video and Signal Based Surveillance pp 574-579, 2010.

[3] Danian Zheng, Yannan Zhao, Jiaxin Wang, "An efficient method of license plate location, Pattern Recognition Letters," ISSN 0167-8655, 10.1016/j.patrec.2005.04.014. Vol. 26/15, pp 2431-2438, Nov. 2005,

[4] Preemon Rattanathammawat, Thanarat H. Chalidabhongse, "A Car Plate Detector using Edge Information", in proc. International Symposium on Communications and Information Technologies, ISCIT '06., pp.1039-1043, October 18-September 20, 2006.

[5] Xifan Shi, Weizhong Zhao, and Yonghang Shen, "Automatic License Plate Recognition System Based on Color ImageProcessing", Lecture Notes on Computer Science, Springer-Verlag, Vol. 3483, pp. 307-314, 2005.

[6] E.R. Lee, P.K. Kim, H.J. Kim, "Automatic recognition of a car license plate using color image processing", in proc. IEEE International Conference on Image Processing, pp. 301-305, Austin, Texas, 1994.

[7] X. He, H. Zhang, W. Jia, Q. Wu, T. Hintz, ,Combining Global and Local Features for Detection of License Plates in a Video"e, in proc. of Image and Vision Computing New Zealand 2007, pp. 288-293, Hamilton, New Zealand, December 2007

[8] Otsu, N., A threshold selection method from gray-level histogram, in journal on IEEE Transactions on Systems Man and Cybernetics, SMC-9, pp.62-66. 1979.

[9] Qadri, M. T. and Asif, M. Automatic Number Plate Recognition System For Vehicle Identification Using Optical Character Recognition; 2009 in proc. 


\section{International Journal of Science and Research (IJSR) \\ ISSN (Online): 2319-7064}

Index Copernicus Value (2013): 6.14 | Impact Factor (2014): 5.611

International Conference on Education Technology and Computer. IEEE Xplore; pp.335-338. 2009.

[10] Saleh, Marwan D.; Mellah, H.; Mueen, Ahmed; Salih, N. D.; , "An efficient method for vehicle license plate xtraction," International Symposium on Information Technology, 2008. ITSim 2008, vol.2, no., pp.1-5, 26-28 Aug. 2008.

[11]B. Fröba, and A. Ernst, "Face Detection with the Modified Census Transform", in proc. Sixth International Conference on Automatic Face and Gesture Recognition (FGR'04), pp. 91-96, Seoul, Korea, May 17-19, 2004.

[12] Babu, C.N.K.; Nallaperumal, K., "A license plate localization using morphology and recognition," India Conference, 2008. INDICON 2008. Annual IEEE, vol.1, no., pp.34-39, 11-13 Dec. 2008.

[13] Wei-Hsun Lee, Shian-Shyong Tseng, Ching-Hung Wang, "Design and implementation of electronic toll collection system based on vehicle positioning system techniques" in Journal on Computer Communications, Elsevier Science Publishers B. V. Amsterdam, The Netherlands, Vol. 31/12, pp 2925-2933 July 2008.

[14] Faradji, F.; Rezaie, A.H.; Ziaratban, M.; "A Morphological-Based License Plate Location", in proc. IEEE International Conference on ImageProcessing, 2007, Vol. 1, pp:1 - 57 - 1-60, 2007.

[15] Christos-Nikolaos E. Anagnostopoulos, Ioannis E. Anagnostopoulos Ioannis D. Psoroulas, Vassili Loumos, Eleftherios Kayafas, "License Plate Recognition From Still Images and Video Sequences: A Survey" in journal on IEEE transactions on intelligent transportation systems, vol. 9, no. 3, 377-383 September 2008. 